\title{
LIGA-Technology for the fabrication of positioned planar structures
}

\author{
H. Schift, J. Söchtig
}

\begin{abstract}
A fabrication process is presented for polymer inserts in micro-optical benches, which combine the mechanical precision of the LIGA-process with the wide variety of optical functions offered by diffractive optical elements. For this purpose metal masters with continuous relief elements and LIGA-structures were used in a combined molding tool and precision micro-optical elements were replicated by injection molding.
\end{abstract}

\section{1}

\section{Introduction}

The mutual alignment of optical elements such as fibers, lenses, detectors and light sources with sub-micron precision is one of the main tasks of free-space micro-optical set ups, because even small misalignments lead to a significant decrease of coupling efficiencies of optical beams. Anisotropic wet etching of silicon or Deep X-Ray Lithography (DXRL) in polymers have already been used for the fabrication of micro-optical benches, where features such as grooves or pins allow the reproducible positioning of ball lenses and fibers without time consuming manual alignment (see Bauer et al. (1995), Müller et al. (1996)). For elements made by surface structuring a passive alignment is only possible if $3 \mathrm{D}$-structures are added corresponding to those of the optical platform (see Fig. 1).

\section{2}

\section{Diffractive optical elements}

At the Paul Scherrer Institute various micro-optical elements are fabricated using an advanced lithographic fabrication

Received: 25 August 1997/Accepted: 22 September 1997

H. Schift, J. Söchtig

Paul Scherrer Institute, CH-5232 Villigen PSI, Switzerland

Correspondence to: H. Schift

We like to express our thanks to Prof. W. Ehrfeld and his LIGA-team at the Institute of Microtechnology Mainz (IMM), Germany, where much of the work has been done in the program "Microfabrication Capability and Expertise for User Defined Purposes". The work profited much from recent experience with injection molding of miniaturized gear boxes (see Thürigen et al. (1996)). Special thanks to R. Dinges, M. Hörr, R. Lüttge, G. Reuter, S. Schirm and C. Thürigen for their valuable support! Furthermore the work of F. Glaus, J. Pedersen, M. Rossi, H. Schütz, R. Stutz, A. Vonlanthen and S. Westenhöfer of PSI is greatfully acknowledged for the laser writing of the DOEs and the fabrication of the metal shims. This work was partially supported by the European Union in the framework of the HCM II program and funded by the Swiss Bundesamt für Bildung und Wissenschaft (BBW) under project \#94.0167. process. Direct laser writing allows to structure continuous reliefs by varying locally the irradiation dose of a focused laser beam during scanning across a photoresist (see Fig. 2, Gale et al. (1994)). Diffractive lenslets and lenslet arrays, as well as refractive microlens arrays, holograms and other diffractive optical elements (DOE) can be fabricated, which could be used for light forming or imaging functions in optical microsystems. The current limits for usable diffractive lenslets are a maximum relief depth of about $5 \mu \mathrm{m}$ and a minimum segment size of about $5 \mu \mathrm{m}$ (see Herzig et al. (1997)). This makes it possible to generate high numerical aperture lenslets necessary for the coupling of laser light into optical fibers with high efficiency (see Rossi et al. (1995)).

Different DOEs were designed for various coupling schemes like fiber to fiber and laser to fiber coupling. A patch of $250 \mu \mathrm{m}$ was chosen to adjust the micro-optical bench inserts to commercial fiber ribbons with an array of 8 single mode fibers (see Fig. 2).

\section{Fabrication of a combined molding tool}

For the fabrication of transparent polymer inserts a mold was fabricated containing both 3D-positioning structures and planar optical elements made by surface structuring. A metal frame is confined between two aligned metal plates, which forms a micro cavity for injection molding (see Fig. 3 ). The outlines of the frame constitute those of the polymer insert, while the surfaces of the plates define its optical function. Metal frames with a cavity outline dimension of $5 \times 3 \mathrm{~mm}^{2}$ and

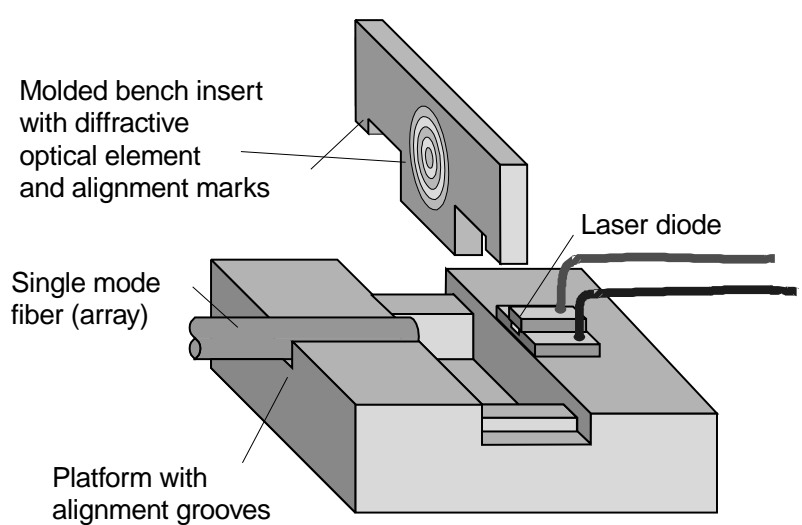

Fig. 1. Platform for laser-fiber coupling with transparent insert containing lens and alignment structures 

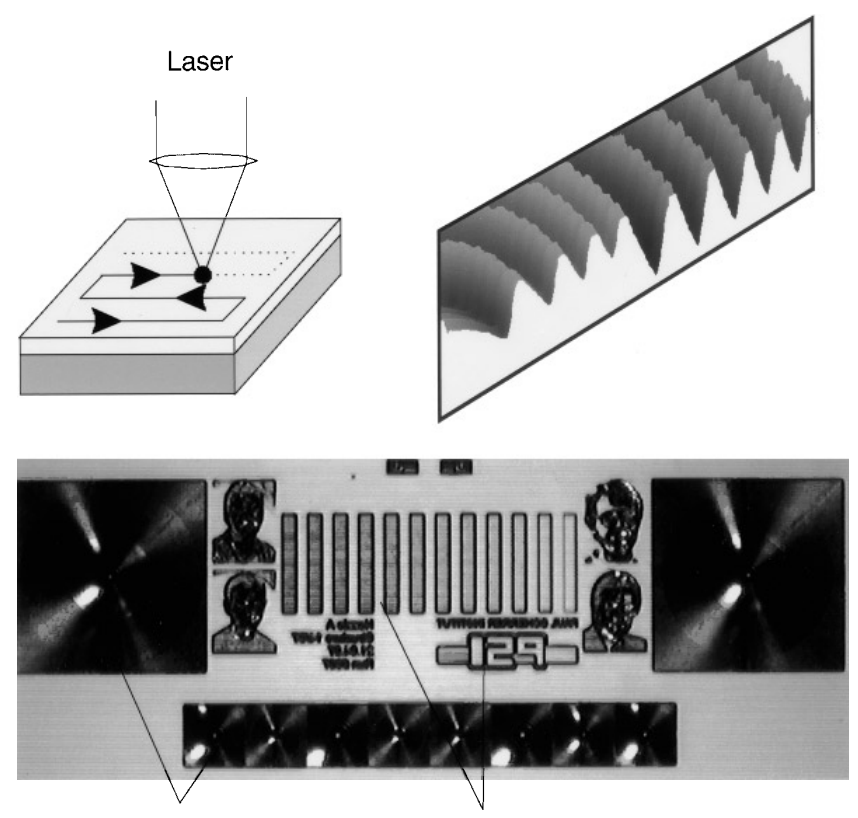

Lenses and test patterns

Fig. 2. Fabrication of a continuous surface relief by direct laser writing in resist (above) and electroplating. The shim (below) contains different lenses and test patterns

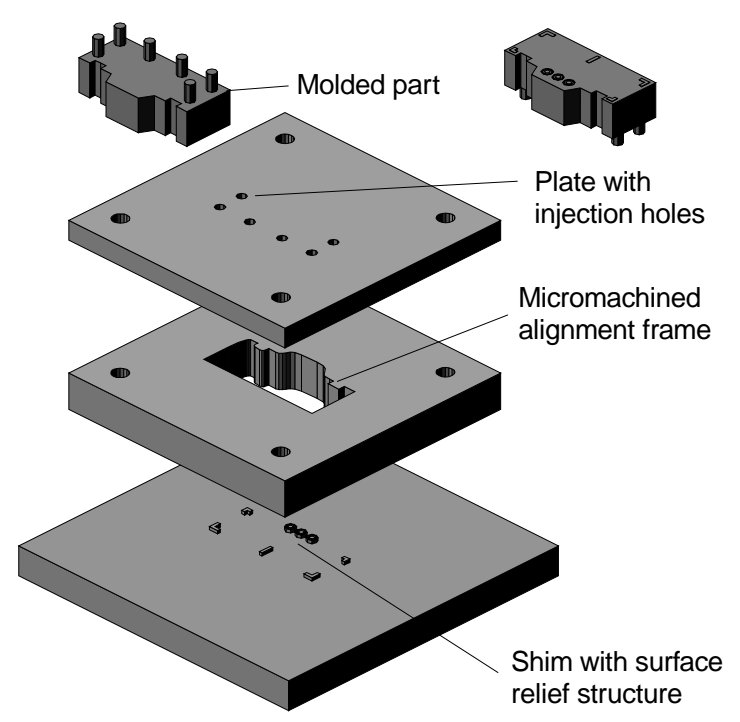

Fig. 3. Schematic drawing of the mold for an injection molding process. The picture shows the three parts constituting the mold cavity

a height of $500 \mu \mathrm{m}$ were fabricated using DXRL and electroplating. Structures with a $\mu \mathrm{m}$ accuracy of the outlines and an excellent surface roughness were obtained, the latter being indispensable for good demolding properties in a replication process. One of the cavity boundaries consisted of a flat $\mathrm{Ni}$-shim with DOEs. Alignment marks and test patterns were also included into the design. The opposite plate of the mold cavity contains the injection nozzle and was defined by

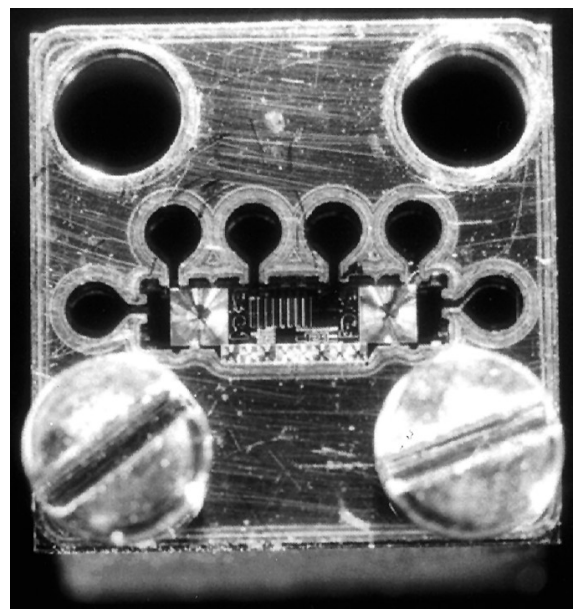

Fig. 4. Mounted mold insert with LIGA metal frame and planar optical element plate. The frame is optically aligned with respect to the lenses and fixed with screws. Size: $7 \times 7 \mathrm{~mm}^{2}$

a polished metal block with a pattern of drilled holes which serve as channels for polymer injection. The pattern of these channels was chosen to achieve a good filling behaviour and assuring demolding without distortion.

The shim was then aligned with respect to the metal LIGA-structures (see Fig. 4) by adjusting the distances of the alignment signs to the borders of the frame under a microscope and was fixed by screws. With this technique a precision of alignment in the $\mu \mathrm{m}$-range could be achieved.

The compact mold insert was then built into the closing unit of a commercial injection molding machine (Aarburg Allrounder 370C) opposite to the injection nozzle.

\section{4}

\section{Replication of polymer inserts with optical elements}

At the beginning of the injection process the nozzle is pressed onto the mold insert which closes the mold cavity. Then the cavity is evacuated and filled with the polymer melt, which solidifies in the cavity. At the end of the process the polymer parts are demolded automatically and separated from the nozzle. Average cycle times are in the order of less than one minute plus separation. Initial experiments have been done using polyethylene (PE), a relatively soft polymer with good demolding properties. The demolded part clearly reproduces the surface structure of all boundaries of the cavity (see Fig. 5a), including diffractive lenses on the front side and alignment posts at the side wall, but is not yet optimized with respect of precision of alignment and filling of the cavity. Furthermore PE as a semi-crystalline material is nontransparent and shows a linear shrinkage of up to $1 \%$. These experiments helped to optimize the mold inserts and the pattern of the injection nozzle. Based on that, further experiments were performed using polycarbonate (PC), an amorphous polymer with excellent optical and mechanical properties (see Fig. 5b). The temperature at the nozzle was about $275^{\circ} \mathrm{C}$ and of the molding tool $120^{\circ} \mathrm{C}$, the injection pressure about 2000 bar. In contrast to PE it was much more critical to demold the structures without distorsion due to the 

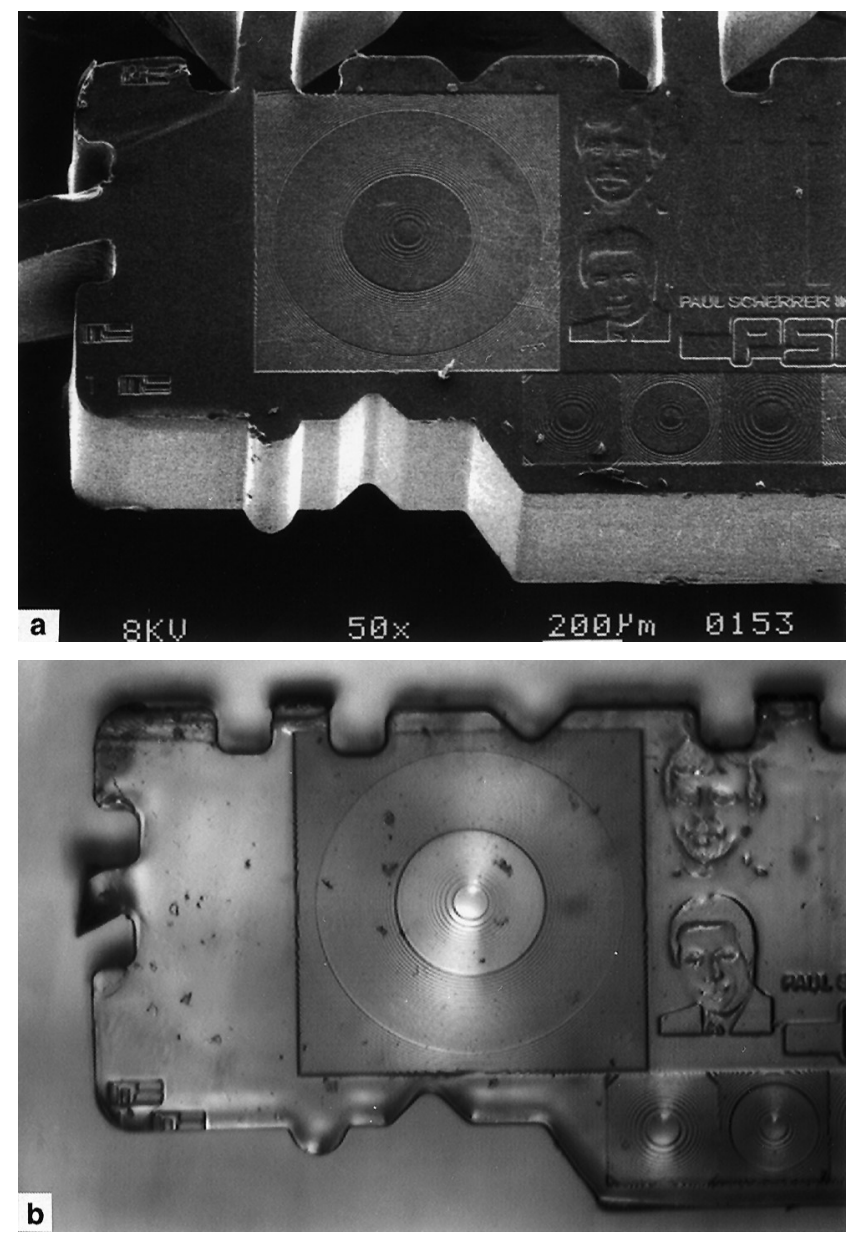

Fig. 5. a SEM pictures of a molded bench insert in polyethylene. The outlines are defined by the LIGA-frame and show typical alignment features at the bottom; $\mathbf{b}$ picture of a molded bench insert in polycarbonate

low shrinkage of the polymer in the cavity. Nevertheless, no anti-adhesives had to be used. Measurements reveiled a linear shrinkage in the order of $0.2 \%$ in the horizontal direction of the elements. Several designs of bench inserts were used, in order to control the shrinkage of the molded element, to restrict damages of the demolding in the vicinity of the injection points and to avoid inhomogeneities in the area of the lenses due to flow anisotropies and seam lines. With careful adjustment of the position and the size of the injection channels these items could be controlled to a large extent.

\section{5}

\section{Summary}

We were able to successfully replicate LIGA structures with aligned DOE structures on the surface by injection molding. The alignment precision lies in the order of several $\mu \mathrm{m}$. Initial experiments were performed to demonstrate the positioning of the structures in an optical bench. Further experiments will be carried out with the aim to improve the alignment precision and the molding properties such as filling of the cavity and the demolding. The imaging properties of the replicated optical elements are now under evaluation.

\section{References}

Bauer H-D; Ehrfeld W; Gerner M; Paatzsch T; Picard A; Schift H; Weber L: (1995): Micro-optical elements and optical interconnection components fabricated using the LIGA technique. Proc. Int. Symp. on Microsystems, Intelligent Materials and Robots, Sendai, Japan, Sept. 1995, pp. 33-36

Müller A; Göttert J; Mohr J; Rogner A: Fabrication of stepped micro-optical benches for fibre and free space applications (1996): High aspect ratio microstructure technology (HARMST '95), Karlsruhe, July 1995, Microsystem Technologies, 2 pp. 40-45

Gale MT; Rossi M; Pedersen J; Schütz H: (1994): Fabrication of continuous-relief micro-optical elements by direct laser writing in photoresists. Optical Engineering, 33, pp. 3556-3566

Herzig H-P; (ed.): Micro-Optics (1997): Elements, systems and applications, chapter 4, London: Taylor \& Francis

Rossi M; Bona GL; Kunz RE: (1995): Arrays of anamorphic phase-matched Fresnel elements for diode-to-fiber coupling. Applied Optics, 34, pp. 2483-2488

Thürigen C; Ehrfeld W; Hagemann B; Kämper K-P; Lehr H; Michel F: (1996): Gesichtspunkte beim Miniaturisieren von Umlaufrädergetrieben, Proc. Symp. Innovative Kleinantriebe, Mainz, May 1996, VDI-Bericht 1269 